1 Hacettepe Journal of Mathematics and Statistics

רolume 45 (1) (2016), $107-120$

\title{
$\oplus$-supplemented modules relative to an ideal
}

\author{
Rachid Tribak*, Yahya Talebi ${ }^{\dagger}$, Ali Reza Moniri Hamzekolaee ${ }^{\ddagger}$ and Samira Asgari ${ }^{\S}$
}

\begin{abstract}
Let $I$ be an ideal of a ring $R$ and let $M$ be a left $R$-module. A submodule $L$ of $M$ is said to be $\delta$-small in $M$ provided $M \neq L+X$ for any proper submodule $X$ of $M$ with $M / X$ singular. An $R$-module $M$ is called $I$ - $\oplus$-supplemented if for every submodule $N$ of $M$, there exists a direct summand $K$ of $M$ such that $M=N+K, N \cap K \subseteq I K$ and $N \cap K$ is $\delta$-small in $K$. In this paper, we investigate some properties of $I$ - $\oplus$-supplemented modules. We also compare $I$ - $\oplus$-supplemented modules with $\oplus$-supplemented modules. The structure of $I$ - $\oplus$-supplemented modules and $\oplus$ - $\delta$-supplemented modules over a Dedekind domain is completely determined.
\end{abstract}

Keywords: $\quad \delta$-small submodules, $\oplus$-supplemented modules, $\oplus-\delta$-supplemented modules, $I$ - $\oplus$-supplemented modules

2000 AMS Classification: 16D10, 16D70, 16D99

Received : 16.02.2014 Accepted: 20.05.2014 Doi : 10.15672/HJMS.20164512490

\section{Introduction}

All rings considered in this paper will be associative with an identity element and $R$ will always denote a ring. We shall use $J(R)$ to denote the Jacobson radical of $R$. All modules will be unital left $R$-modules. Let $M$ be an $R$-module. A submodule $L$ of $M$ is called small ( $\delta$-small) in $M$, denoted by $L \ll M(L \ll \delta M)$, if $L+X \neq M$ for any proper submodule $X$ of $M(L+X \neq M$ for any proper submodule $X$ of $M$ with $M / X$ singular). Recall that $M$ is called $\oplus$-supplemented $(\oplus$ - $\delta$-supplemented $)$ if for every submodule $N \leq M$, there exists a direct summand $K$ of $M$ such that $N+K=M$ and $N \cap K \ll K\left(N \cap K \ll_{\delta} K\right)$.

\footnotetext{
*Centre Régional des Métiers de l'Education et de la Formation (CRMEF)-Tanger, Avenue My Abdelaziz, BP 3117 Souani, Tanger, Morocco. Email: tribak12@yahoo.com

${ }^{\dagger}$ Department of Mathematics, Faculty of Mathematical Sciences, University of Mazandaran, Babolsar, Iran. Email: talebi@umz.ac.ir

${ }^{\ddagger}$ Department of Mathematics, Faculty of Mathematical Sciences, University of Mazandaran, Babolsar, Iran. Email: a.monirih@umz.ac.ir

${ }^{\S}$ Department of Mathematics, Islamic Azad University, Mobarakeh Branch, Mobarakeh, Iran. Email: samira.asgari.a@gmail.com
} 
In Section 2, we study some special cases of submodules $N$ of a module $M$ for which $N \ll{ }_{\delta} M$ is equivalent to $N \ll M$.

In Section 3, we introduce the notion of $I$ - $\oplus$-supplemented $R$-modules, where $I$ is an ideal of $R$. A module $M$ will be called $I$ - $\oplus$-supplemented if for every submodule $N$ of $M$, there exists a direct summand $K$ of $M$ such that $M=N+K, N \cap K \subseteq I K$ and $N \cap K \ll_{\delta} K$. We shall compare this notion with the concept of $\oplus$-supplemented modules. Indecomposable $I$ - $\oplus$-supplemented modules are characterized.

Section 4 is devoted to the study of some factor modules of an $I$ - $\oplus$-supplemented module. Among other results, it is shown that if $M$ is a direct sum of two hollow $I$ - $\oplus$-supplemented modules, then any direct summand of $M$ is $I$ - $\oplus$-supplemented.

In Section 5, our main results (Theorems 5.4 and 5.13) describe the structure of $I$ - $\oplus$-supplemented modules over Dedekind domains. It is also shown that over a Dedekind domain $R$, an $R$-module $M$ is $\oplus$ - $\delta$-supplemented if and only if $M$ is $\oplus$-supplemented.

\section{Some properties of $\delta$-small submodules}

We begin with some results presenting some elementary properties of $\delta$-small submodules which will be used in the sequel.

2.1. Lemma. ([19, Lemma 1.2]) Let $N$ be a submodule of a module $M$. The following are equivalent:

(i) $N$ is $\delta$-small in $M$;

(ii) If $X+N=M$, then $M=X \oplus Y$ for a projective semisimple submodule $Y$ with $Y \leq N$.

2.2. Lemma. (See [19, Lemma 1.3])

(i) Let $N$ and $K$ be submodules of a module $M$ with $K \subseteq N$. If $N \ll_{\delta} M$, then $K \ll_{\delta} M$.

(ii) Let $M$ and $M^{\prime}$ be two modules. If $L \ll \delta M$ and $f: M \rightarrow M^{\prime}$ is a homomorphism, then $f(L) \ll_{\delta} M^{\prime}$. In particular, if $K \ll_{\delta} M \leq M^{\prime}$, then $K \ll_{\delta} M^{\prime}$.

(iii) If $N$ and $L$ are submodules of a module $M$, then $N+L \ll \delta M$ if and only if $N \ll \delta M$ and $L \ll_{\delta} M$.

(iv) Let $M_{1}$ and $M_{2}$ be two submodules of a module $M$ such that $M=M_{1} \oplus M_{2}$. Let $K_{1} \leq M_{1}$ and $K_{2} \leq M_{2}$. Then $K_{1} \oplus K_{2} \ll_{\delta} M_{1} \oplus M_{2}$ if and only if $K_{1} \ll_{\delta} M_{1}$ and $K_{2} \ll_{\delta} M_{2}$.

Let $N$ be a submodule of a module $M$. Recall that $N$ is said to be $D M$ in $M$ (or $N$ decomposes $M)$ if there is a direct summand $D$ of $M$ such that $D \leq N$ and $M=D+X$, whenever $N+X=M$ for a submodule $X$ of $M$ (see [1, Definition 3.1]). Clearly, the following implications hold:

$$
(N \ll M) \Rightarrow(N \ll \delta M) \Rightarrow(N \text { is } D M \text { in } M) .
$$

Next, we exhibit some conditions under which $N \ll \delta M$ is equivalent to $N \ll M$.

2.3. Proposition. Let $N$ be a proper submodule of an indecomposable module $M$. Then $N$ is $D M$ in $M$ if and only if $N \ll \delta$ if and only if $N \ll M$.

Proof. Assume that $N$ is $D M$ in $M$. Let $X$ be a submodule of $M$ such that $M=N+X$. Then there exists a direct summand $D$ of $M$ such that $D \leq N$ and $M=D+X$. Since $M$ is indecomposable and $N \neq M$, we have $D=0$ and $X=M$. Therefore, $N \ll M$. The rest of the proof is immediate.

The next result was inspired by [16, Proposition 2.3(1)].

2.4. Proposition. Let $N$ be a submodule of a module $M$. Then $N \ll M$ if and only if $N \subseteq \operatorname{Rad}(M)$ and $N \ll \delta M$.

Proof. It is enough to prove the sufficiency. Let $X$ be a submodule of $M$ such that $M=N+X$. Since $N \ll_{\delta} M$, there exists a projective semisimple submodule $P \leq N$ such that $M=P \oplus X$. 
Assume that $P \neq 0$. Then $P$ has a simple direct summand $S$. Since $S \subseteq \operatorname{Rad}(M), S \ll M$. Hence $S=0$, a contradiction. Thus, $P=0$. It follows that $N \ll M$.

The following result is a direct consequence of Proposition 2.4.

2.5. Corollary. Let $M$ be a module with $\operatorname{Rad}(M)=M$ and let $N$ be a submodule of $M$. Then $N \ll \delta$ if and only if $N \ll M$.

Let $M$ be a module over a commutative integral domain $R$. Let $T(M)$ denote the set of all elements $x \in M$ for which there exists a nonzero element $r \in R$ such that $r x=0$. It is well known that $T(M)$ is a submodule of $M$. This submodule is called the torsion submodule of $M$. If $T(M)=M$, then the module $M$ is said to be a torsion module. The module $M$ is said to be torsion-free if $T(M)=0$.

2.6. Proposition. Assume that $R$ is a commutative integral domain. Let $M$ be an $R$-module and $N$ a submodule of $M$ such that $N \subseteq T(M)$. Then $N \ll_{\delta} M$ if and only if $N \ll M$.

Proof. Assume that $N \ll \delta M$. Let $X$ be a submodule of $M$ such that $N+X=M$. Then there exists a projective submodule $P \leq N$ such that $P \oplus X=M$. Since $P$ is projective, $P$ is isomorphic to a direct summand of a free $R$-module. Hence, $P$ is torsion-free. But $P$ is a torsion module as $P \subseteq N$. Then $P=0$ and $X=M$. It follows that $N \ll M$. The converse is obvious.

Let $N$ and $K$ be submodules of a module $M$. Recall that $K$ is said to be a supplement of $N$ in $M$ if $N+K=M$ and $N \cap K \ll K$. Let $M=\oplus_{i \in I} M_{i}$ be a decomposition of the module $M$. The next example shows that, in general, if $L=\bigoplus_{i \in I} L_{i}$ is a submodule of $M$ such that $L_{i} \ll_{\delta} M_{i}$ for each $i \in I$, then $L$ need not be $\delta$-small in $M$.

2.7. Example. Let $R$ be a discrete valuation ring with maximal ideal $m$. Let $M=\oplus_{i=1}^{\infty} R / m^{i}$. By [20, p. 48 The second corollary of Lemma 2.1], $\operatorname{Rad}(M)$ does not have a supplement in $M$. Therefore, $\operatorname{Rad}(M)=\oplus_{i=1}^{\infty} m / m^{i}$ is not small in $M$. Applying Proposition 2.6, it follows that $\operatorname{Rad}(M)$ is not $\delta$-small in $M$. On the other hand, it is clear that for each $i \geq 1, m / m^{i} \ll R / m^{i}$.

2.8. Proposition. Let $M=\oplus_{i \in I} M_{i}$ be a decomposition of a module $M$. Assume that for every submodule $N \leq M$, we have $N=\oplus_{i \in I}\left(N \cap M_{i}\right)$. For each $i$, let $L_{i}$ be a submodule of $M_{i}$. The following statements are equivalent:

(i) $L_{i} \ll_{\delta} M_{i}$ for every $i \in I$;

(ii) $L=\oplus_{i \in I} L_{i} \ll \delta M$.

Proof. (i) $\Rightarrow$ (ii) Let $X$ be a submodule of $M$ such that $M=X+L$. By hypothesis, $X=$ $\oplus_{i \in I}\left(X \cap M_{i}\right)$. So, $\left(X \cap M_{i}\right)+L_{i}=M_{i}$ for every $i \in I$. By assumption, for every $i \in I$, there exists a semisimple projective submodule $P_{i}$ of $L_{i}$ such that $\left(X \cap M_{i}\right) \oplus P_{i}=M_{i}$ (see Lemma 2.1). Let $P=\oplus_{i \in I} P_{i}$. Then $X \oplus P=M$. Note that $P$ is a semisimple projective submodule of $L$. Therefore, $L \ll \delta M$.

(ii) $\Rightarrow$ (i) By Lemma 2.2(iv).

\section{3. $I$ - $\oplus$-supplemented modules}

Recall that a module $M$ is called $\oplus$-supplemented $(\oplus-\delta$-supplemented) if for every submodule $N \leq M$, there exists a direct summand $K$ of $M$ such that $N+K=M$ and $N \cap K \ll K$ $\left(N \cap K \ll_{\delta} K\right)$.

Recall that a ring $R$ is said to be semilocal provided $R / J(R)$ is a semisimple ring. 
3.1. Proposition. Let $M$ be a module over a semilocal ring $R$. Then $M$ is $\oplus$-supplemented if and only if for every submodule $N \leq M$, there exists a direct summand $K$ of $M$ such that $M=N+K$, $N \cap K \subseteq J(R) K$ and $N \cap K \ll \delta K$.

Proof. By Proposition 2.4 and [2, Corollary 15.18].

Motivated by the last proposition, we introduce the following notion:

3.2. Definition. Let $M$ be an $R$-module and let $I$ be an ideal of $R$. We say that $M$ is $I$ - $\oplus$ supplemented, provided for every submodule $N$ of $M$, there exists a direct summand $K$ of $M$ such that $M=N+K, N \cap K \subseteq I K$ and $N \cap K \ll \delta K$.

In this section we investigate some properties of $I$ - $\oplus$-supplemented modules.

3.3. Remark. (i) It is clear that for every ideal $I$ of $R$, every $I$ - $\oplus$-supplemented module is $\oplus-\delta$ supplemented.

(ii) Let $M$ be an $R$-module. If $I$ is an ideal of $R$ such that $I M=0$, then $M$ is $I$ - $\oplus$-supplemented if and only if $M$ is semisimple.

Let $M$ be an $R$-module. As in [19], let $\delta(M)$ denote the sum of all $\delta$-small submodules of $M$. In the next proposition we provide a condition under which a $\oplus$ - $\delta$-supplemented module is $I$ - $\oplus$-supplemented. To prove this result, we need the following elementary lemma.

3.4. Lemma. Let $M$ be an $R$-module and let $I$ be an ideal of $R$. If $K$ is a direct summand of $M$, then we have $I K=K \cap I M$.

Proof. Let $K^{\prime}$ be a submodule of $M$ such that $M=K \oplus K^{\prime}$. Then $I M=I K \oplus I K^{\prime}$. Hence $K \cap I M=I K$.

3.5. Proposition. Let $M$ be an $R$-module and let $I$ be an ideal of $R$ such that $\delta(M) \subseteq I M$. Then $M$ is $I$ - $\oplus$-supplemented if and only if $M$ is $\oplus$ - $\delta$-supplemented.

Proof. The necessity is clear. Conversely, suppose that $M$ is $\oplus$ - $\delta$-supplemented. Let $N$ be a submodule of $M$. Then there exists a direct summand $K$ of $M$ such that $M=N+K$ and $N \cap K \ll_{\delta} K$. Note that $I K=K \cap I M$ by Lemma 3.4. Since $\delta(M) \subseteq I M$, we have

$$
N \cap K \subseteq \delta(K) \subseteq K \cap \delta(M) \subseteq K \cap I M=I K .
$$

Therefore $M$ is $I$ - $\oplus$-supplemented. This completes the proof.

Recall that a nonzero module $M$ is called hollow if every proper submodule is small in $M$. The module $M$ is called local if it has a proper submodule which contains all other proper submodules. Note that the largest proper submodule of a local module $M$ is $\operatorname{Rad}(M)$. It is well known that every hollow module is $\oplus$-supplemented.

3.6. Example. (i) It is clear that every semisimple module is $I$ - $\oplus$-supplemented for any ideal $I$ of $R$.

(ii) Let $p$ be a prime integer. It is well known that the $\mathbb{Z}$-module $\mathbb{Z}\left(p^{\infty}\right)$ is hollow and injective. It is easily seen that $\mathbb{Z}\left(p^{\infty}\right)$ is $I$ - $\oplus$-supplemented for every nonzero ideal $I$ of $\mathbb{Z}$, but $\mathbb{Z}\left(p^{\infty}\right)$ is not 0 - $\oplus$-supplemented.

(iii) It is easy to see that every $\oplus$ - $\delta$-supplemented module (in particular, every $\oplus$-supplemented module) is $R$ - $\oplus$-supplemented (see Proposition 3.5).

3.7. Proposition. Let $M$ be an indecomposable $R$-module and let $I$ be an ideal of $R$. The following conditions are equivalent:

(i) $M$ is $I$ - $\oplus$-supplemented;

(ii) $M$ is hollow with $I M=M$ or $I M=\operatorname{Rad}(M)$. 
Proof. (i) $\Rightarrow$ (ii) Let $N$ be a proper submodule of $M$. By hypothesis, there exists a direct summand $K$ of $M$ such that $N+K=M, N \cap K \subseteq I K$ and $N \cap K \ll_{\delta} K$. Since $M$ is indecomposable, we have $K=M$. Hence, $N \subseteq I M$ and $N \ll \delta M$. By Proposition 2.3, we have $N \ll M$. Thus, $M$ is a hollow module. Moreover, note that if $I M \neq M$, then $I M$ contains all other proper submodules of $M$. Hence $M$ is a local module and $I M=\operatorname{Rad}(M)$.

(ii) $\Rightarrow$ (i) Let $N$ be a proper submodule of $M$. Then $N+M=M, N \cap M=N \subseteq \operatorname{Rad}(M) \subseteq I M$ and $N \cap M=N \ll_{\delta} M$. Therefore, $M$ is $I$ - $\oplus$-supplemented.

It follows from Proposition 3.7 that if $I$ is an ideal of $R$, then every indecomposable $I$ - $\oplus$ supplemented $R$-module is $\oplus$-supplemented. Next, we present some examples of $\oplus$-supplemented modules which are not $I$ - $\oplus$-supplemented for an ideal $I$ of $R$.

3.8. Example. (i) Let $p$ and $q$ be two different prime integers. Consider the local $\mathbb{Z}$-module $M=$ $\mathbb{Z} / \mathbb{Z} p^{3}$. We have $\operatorname{Rad}(M)=\mathbb{Z} p / \mathbb{Z} p^{3}$. Let $I_{1}=\mathbb{Z} p, I_{2}=\mathbb{Z} q$ and $I_{3}=\mathbb{Z} p^{2}$. Then $I_{1} M=\operatorname{Rad}(M)$, $I_{2} M=M$ and $I_{3} M=\mathbb{Z} p^{2} / \mathbb{Z} p^{3}$. By Proposition 3.7, $M$ is $I_{i}$ - $\oplus$-supplemented for each $i=1,2$, but not $I_{3}-\oplus$-supplemented. On the other hand, it is clear that $M$ is $\oplus$-supplemented.

(ii) Let $R$ be a discrete valuation ring with maximal ideal $m$. It is well known that the $R$-module ${ }_{R} R$ is $\oplus$-supplemented. Let $I$ be an ideal of $R$. From Proposition 3.7 it follows that ${ }_{R} R$ is $I$ - $\oplus$ supplemented if and only if $I=m$ or $I=R$. Therefore, the module ${ }_{R} R$ is not $m^{3}-\oplus$-supplemented.

3.9. Proposition. Let $I$ be an ideal of $R$ and let $M$ be an $R$-module.

(i) Assume that for every submodule $N \leq M$, there exists a submodule $K \leq M$ such that $M=N+K$ and $N \cap K \subseteq I M$. Then $M / I M$ is semisimple.

(ii) If $M$ is an $I$ - $\oplus$-supplemented $R$-module, then $M / I M$ is semisimple.

Proof. (i) Let $N$ be a submodule of $M$ such that $I M \subseteq N$. By assumption, there exists a submodule $K$ of $M$ such that $N+K=M$ and $N \cap K \subseteq I M$. Thus, $(N / I M)+[(K+I M) / I M]=M / I M$. Clearly, we have $N \cap(K+I M)=I M$. So, $N / I M$ is a direct summand of $M / I M$. This completes the proof.

(ii) follows from (i).

3.10. Proposition. Let $M$ be a module.

(i) If $M$ is $\oplus$ - $\delta$-supplemented, then $M=M_{1} \oplus M_{2}$ such that $\operatorname{Rad}\left(M_{1}\right) \ll M_{1}$ and $\operatorname{Rad}\left(M_{2}\right)=M_{2}$.

(ii) If $M$ is $I$ - $\oplus$-supplemented, then $M=M_{1} \oplus M_{2}$ such that $\operatorname{Rad}\left(M_{1}\right) \subseteq I M_{1}, \operatorname{Rad}\left(M_{1}\right) \ll M_{1}$ and $\operatorname{Rad}\left(M_{2}\right)=M_{2}$.

Proof. (i) Since $M$ is $\oplus$ - $\delta$-supplemented, there exist submodules $M_{1}$ and $M_{2}$ of $M$ such that $M=$ $M_{1} \oplus M_{2}, \operatorname{Rad}(M)+M_{1}=M$ and $\operatorname{Rad}(M) \cap M_{1} \ll \delta M_{1}$. Note that $\operatorname{Rad}(M)=\operatorname{Rad}\left(M_{1}\right) \oplus \operatorname{Rad}\left(M_{2}\right)$. Then $M_{1} \oplus \operatorname{Rad}\left(M_{2}\right)=M$ and $\left(\operatorname{Rad}(M) \cap M_{1}\right) \oplus \operatorname{Rad}\left(M_{2}\right)=\operatorname{Rad}(M)$. Therefore $\operatorname{Rad}\left(M_{2}\right)=M_{2}$ and $\operatorname{Rad}(M) \cap M_{1}=\operatorname{Rad}\left(M_{1}\right)$. Moreover, we have $\operatorname{Rad}\left(M_{1}\right) \ll M_{1}$ by Proposition 2.4. This completes the proof.

(ii) This follows by the same method as in (i) and adding the fact that $\operatorname{Rad}(M) \cap M_{1} \subseteq I M_{1}$.

Combining Proposition 3.10(ii) and [2, Proposition 5.20(1)], we get the following result.

3.11. Corollary. If $M$ is an $I$ - $\oplus$-supplemented module with $\operatorname{Rad}(M) \ll M$, then $\operatorname{Rad}(M) \subseteq I M$.

From the last corollary, we conclude that if $I$ is an ideal of a left perfect ring $R$ and $M$ is an $I$ - $\oplus$-supplemented $R$-module, then $\operatorname{Rad}(M) \subseteq I M$ (see [2, Remark 28.5(3)]).

An $R$-module $M$ is said to be $\delta$-local if $\delta(M) \ll \delta M$ and $\delta(M)$ is a maximal submodule of $M$ (see [4, Definition 3.1]). Next, we give an example of an $R$ - $\oplus$-supplemented module which is not $\oplus$-supplemented. 
3.12. Example. Let $F=\mathbb{Z} / \mathbb{Z} 2$ and let $A=F^{\mathbb{N}}$ be the ring of sequences over $F$, whose operations are pointwise multiplication and pointwise addition. Let $R \subseteq A$ be the subring generated by $1_{A}$ (the unit element of $A$ ) and all sequences that have only a finite number of nonzero entries. It is shown in [4, p. 318] that the $\operatorname{ring} R$ is not semilocal and the $R$-module ${ }_{R} R$ is $\delta$-local. Applying [15, Proposition 3.1], it is easily seen that ${ }_{R} R$ is an $R-\oplus$-supplemented module. On the other hand, since the ring $R$ is not semilocal, it is not semiperfect. Hence, the $R$-module ${ }_{R} R$ is not $\oplus$-supplemented by [12, Corollary 4.42].

Next, we present conditions under which an $I$ - $\oplus$-supplemented $R$-module is $\oplus$-supplemented.

3.13. Proposition. Let $M$ be an $R$-module with $\operatorname{Rad}(M)=M$. Then $M$ is $\oplus$ - $\delta$-supplemented if and only if $M$ is $\oplus$-supplemented.

Proof. As $\operatorname{Rad}(M)=M$, we have $\operatorname{Rad}(K)=K$ for every direct summand $K$ of $M$. The result follows from Corollary 2.5.

3.14. Proposition. Assume that $R$ is a commutative integral domain and let $M$ be a torsion $R$-module. Then $M$ is $\oplus-\delta$-supplemented if and only if $M$ is $\oplus$-supplemented.

Proof. This follows from Proposition 2.6.

3.15. Proposition. Let $I$ be an ideal of $R$ and let $M$ be an $I$ - $\oplus$-supplemented $R$-module. If $I M \subseteq \operatorname{Rad}(M)$, then $M$ is $\oplus$-supplemented.

Proof. Let $N$ be a submodule of $M$. By hypothesis, there exists a direct summand $K$ of $M$ such that $M=N+K, N \cap K \subseteq I K$ and $N \cap K \ll_{\delta} K$. Since $I M \subseteq \operatorname{Rad}(M)$, we have $I K=K \cap I M \subseteq K \cap \operatorname{Rad}(M)=\operatorname{Rad}(K)$ by Lemma 3.4 and [5, 20.4(7)]. So $N \cap K \ll K$ by Proposition 2.4. It follows that $M$ is $\oplus$-supplemented.

3.16. Corollary. Let $I$ be an ideal of $R$ and let $M$ be an $I$ - $\oplus$-supplemented $R$-module. Assume that one of the following conditions is satisfied:

(i) $I \subseteq J(R)$, or

(ii) $R$ is a local ring and $I \neq R$, or

(iii) $\operatorname{Rad}(M)=M$, or

(iv) $R$ is a commutative integral domain and $M$ is a torsion $R$-module.

Then $M$ is $\oplus$-supplemented.

Proof. (i) follows from [2, Corollary 15.18] and Proposition 3.15.

(ii) follows from (i)

(iii) follows easily from Proposition 3.13.

(iv) is obvious by Proposition 3.14.

Next, we focus on when a $\oplus$-supplemented $R$-module is $I$ - $\oplus$-supplemented for an ideal $I$ of $R$.

3.17. Proposition. Let $I$ be an ideal of $R$ and let $M$ be $a \oplus$-supplemented $R$-module such that $\operatorname{Rad}(M) \subseteq I M$. Then $M$ is $I-\oplus$-supplemented.

Proof. Let $N$ be a submodule of $M$. Then there exists a direct summand $K$ of $M$ such that $M=N+K$ and $N \cap K \ll K$. Thus, $N \cap K \ll_{\delta} K$. Moreover, we have $I K=K \cap I M$ by Lemma 3.4. Since $\operatorname{Rad}(M) \subseteq I M$, it follows that

$$
\operatorname{Rad}(K) \subseteq K \cap \operatorname{Rad}(M) \subseteq K \cap I M=I K .
$$

Hence, $N \cap K \subseteq I K$. Therefore $M$ is $I$ - $\oplus$-supplemented. This completes the proof.

The next corollary is a direct consequence of Proposition 3.17. 
3.18. Corollary. Let $M$ be a $\oplus$-supplemented module such that $I M=M$. Then $M$ is $I-\oplus$ supplemented.

3.19. Corollary. Let $m$ be a maximal ideal of a commutative ring $R$ and let $M$ be an $R$-module. Assume that $I$ is an ideal of $R$ such that $I M=m M$. If $M$ is a $\oplus$-supplemented $R$-module, then $M$ is $I$ - $\oplus$-supplemented.

Proof. Note that $\operatorname{Rad}(M) \subseteq m M$ by [7, Lemma 3]. The result follows from Proposition 3.17.

Let $R$ be a commutative integral domain. An $R$-module $M$ is called divisible in case $r M=M$ for each nonzero element $r \in R$.

3.20. Corollary. Let $M$ be a divisible module over a commutative integral domain $R$. If $M$ is $\oplus$-supplemented, then $M$ is $I$ - $\oplus$-supplemented for every nonzero ideal I of $R$.

Proof. This follows from Corollary 3.18.

Recall that a ring $R$ is called a left good ring if $\operatorname{Rad}(M)=J(R) M$ for every $R$-module $M$ (see $[18,23.7])$.

3.21. Corollary. Let $M$ be an R-module. Suppose further that either

(i) $R$ is a left good ring, or

(ii) $M$ is a projective module.

Then $M$ is $\oplus$-supplemented if and only if $M$ is $J(R)-\oplus$-supplemented.

Proof. Note that $\operatorname{Rad}(M)=J(R) M$ by [2, Proposition 17.10]. The result follows from Propositions 3.15 and 3.17.

Combining Lemma 2.2 and the application of the same reasoning of [10, Proposition 3] to $I$ - $\oplus$ supplemented modules, we obtain the following theorem.

3.22. Theorem. Let $I$ be an ideal of $R$. Then any finite direct sum of $I$ - $\oplus$-supplemented $R$-modules is $I$ - $\oplus$-supplemented.

The next example shows that, in general, a direct sum of $I$ - $\oplus$-supplemented modules is not $I$ - $\oplus$-supplemented.

3.23. Example. Let $p$ be a prime integer. Consider the $\mathbb{Z}$-module $M=\oplus_{i=1}^{\infty} \mathbb{Z} / \mathbb{Z} p^{i}$. Clearly, $M$ is a torsion module. By [12, Propositions A.7 and A.8], $M$ is not $\oplus$-supplemented. Therefore $M$ is not $(\mathbb{Z} p)$ - $\oplus$-supplemented by Corollary 3.16. On the other hand, note that for every $i \geq 1, \mathbb{Z} / \mathbb{Z} p^{i}$ is a $(\mathbb{Z} p)$ - $\oplus$-supplemented $\mathbb{Z}$-module by Proposition 3.7 .

The next result deals with a special case of a family of $\oplus$ - $\delta$-supplemented $(I$ - $\oplus$-supplemented) modules $\left(M_{\lambda}\right)_{\lambda \in \Lambda}$ for which $M=\oplus_{\lambda \in \Lambda} M_{\lambda}$ is $\oplus$ - $\delta$-supplemented ( $I$ - $\oplus$-supplemented).

3.24. Proposition. Let $I$ be an ideal of $R$ and let $M=\oplus_{\lambda \in \Lambda} M_{\lambda}$ be a direct sum of submodules $M_{\lambda}(\lambda \in \Lambda)$ such that for every submodule $N$ of $M$, we have $N=\oplus_{\lambda \in \Lambda}\left(N \cap M_{\lambda}\right)$. Assume that $M_{\lambda}$ is $\oplus$ - $\delta$-supplemented ( $I-\oplus$-supplemented) for every $\lambda \in \Lambda$. Then $M$ is $\oplus$ - $\delta$-supplemented $(I-\oplus-$ supplemented).

Proof. Let $N$ be a submodule of $M$. Then $N=\oplus_{\lambda \in \Lambda}\left(N \cap M_{\lambda}\right)$. For every $\lambda \in \Lambda$, there exists a direct summand $K_{\lambda}$ of $M_{\lambda}$ such that $\left(N \cap M_{\lambda}\right)+K_{\lambda}=M_{\lambda},\left(N \cap K_{\lambda} \subseteq I K_{\lambda}\right)$ and $N \cap K_{\lambda} \ll_{\delta} K_{\lambda}$. Set $K=\oplus_{\lambda \in \Lambda} K_{\lambda}$. Clearly, $K$ is a direct summand of $M$ and $N+K=M$. Also, we have $(N \cap K=$ $\left.\oplus_{\lambda \in \Lambda}\left(N \cap K_{\lambda}\right) \subseteq I K\right)$ and $N \cap K \ll_{\delta} K$ by Proposition 2.8. This proves the proposition. 


\section{Homomorphic images of $I$ - $\oplus$-supplemented modules}

We begin this section by an example showing that the $I$ - $\oplus$-supplemented property does not always transfer from a module to each of its factor modules.

4.1. Example. Let $F$ be a field. Consider the local $\operatorname{ring} R=F\left[x^{2}, x^{3}\right] /\left(x^{4}\right)$ and let $m$ be the maximal ideal of $R$. Let $n$ be an integer with $n \geq 2$ and let $M=R^{(n)}$. By Proposition 3.7 and Theorem 3.22, $M$ is $m$ - $\oplus$-supplemented. Note that $R$ is an artinian local ring which is not a principal ideal ring (see [3, Example on p. 91]). So, there exists a submodule $K$ of $M$ such that the factor module $M / K$ is not $\oplus$-supplemented by [11, Example 2.2]. Therefore $M / K$ is not $m$ - $\oplus$-supplemented by Corollary 3.16 .

Next, we show that under some conditions, a factor module of an $I$ - $\oplus$-supplemented module is $I$ - $\oplus$-supplemented.

Recall that a submodule $N$ of a module $M$ is called fully invariant if $f(N) \subseteq N$ for every endomorphism $f$ of $M$. A module $M$ is called distributive if $(A+B) \cap C=(A \cap C)+(B \cap C)$ for all submodules $A, B, C$ of $M$ (or equivalently, $(A \cap B)+C=(A+C) \cap(B+C)$ for all submodules $A, B, C$ of $M)$.

Analysis similar to the proofs of [6, Theorems 4.7 and 4.8] yields the following result. We give the first part of its proof for completeness.

4.2. Proposition. Let $I$ be an ideal of $R$ and let $M$ be an $I$ - $\oplus$-supplemented module.

(i) Let $X \leq M$ be a submodule such that for every direct summand $K$ of $M,(X+K) / X$ is a direct summand of $M / X$. Then $M / X$ is $I$ - $\oplus$-supplemented.

(ii) Let $X \leq M$ be a submodule such that for every decomposition $M=M_{1} \oplus M_{2}$, we have $X=\left(X \cap M_{1}\right) \oplus\left(X \cap M_{2}\right)$. Then $M / X$ is $I-\oplus$-supplemented.

(iii) If $X$ is a fully invariant submodule of $M$, then $M / X$ is $I$ - $\oplus$-supplemented.

(iv) If $M$ is a distributive module, then $M / X$ is $I-\oplus$-supplemented for every submodule $X$ of $M$.

Proof. (i) Let $N$ be a submodule of $M$ such that $X \subseteq N$. Since $M$ is $I$ - $\oplus$-supplemented, there exists a direct summand $K$ of $M$ such that $N+K=M, N \cap K \subseteq I K$ and $N \cap K \ll_{\delta} K$. Therefore $(N / X)+((X+K) / X)=M / X$ and $(N / X) \cap((K+X) / X)=(X+(N \cap K)) / X \subseteq((X+I K) / X) \subseteq$ $I((X+K) / X)$. Consider the natural epimorphism $\pi: K \rightarrow(X+K) / X$. Since $N \cap K \ll{ }_{\delta} K$, we have $\pi(N \cap K)=(X+(N \cap K)) / X \ll_{\delta}(X+K) / X$ by Lemma 2.2(ii). Note that by assumption, $(X+K) / X$ is a direct summand of $M / X$. It follows that $M / X$ is $I$ - $\oplus$-supplemented.

(ii), (iii) and (iv) These are consequences of (i).

The next proposition was inspired by [11, Proposition 2.5].

4.3. Proposition. Let $M$ be an $R$-module and let $I$ be an ideal of $R$. Let $K$ be a fully invariant direct summand of $M$. Then the following assertions are equivalent:

(i) $M$ is $I$ - $\oplus$-supplemented;

(ii) $K$ and $M / K$ are $I-\oplus$-supplemented.

Proof. (i) $\Rightarrow$ (ii) Let $L$ be a submodule of $K$. By hypothesis, there exist submodules $A$ and $B$ of $M$ such that $M=A \oplus B, M=A+L, A \cap L \subseteq I A$ and $A \cap L \ll_{\delta} A$. Clearly, we have $K=(A \cap K)+L$. Since $K$ is fully invariant in $M$, we have $K=(A \cap K) \oplus(B \cap K)$. Hence, $A \cap K$ is a direct summand of $M$. Thus $I(A \cap K)=(A \cap K) \cap I M$ by Lemma 3.4. It follows that $(A \cap K) \cap L=A \cap L \subseteq(A \cap K) \cap I M=I(A \cap K)$. Since $A \cap L \ll_{\delta} A$ and $A \cap K$ is a direct summand of $A$, we have $A \cap L \ll \delta A \cap K$ by Lemma 2.2(iv). Therefore, $K$ is $I$ - $\oplus$-supplemented. Moreover, $M / K$ is $I$ - $\oplus$-supplemented by Proposition 4.2 (iii).

(ii) $\Rightarrow$ (i) This follows from Theorem 3.22 . 
Let $I$ be an ideal of $R$. An $R$-module $M$ is called completely $I$ - $\oplus$-supplemented $(\oplus$-supplemented) if every direct summand of $M$ is $I$ - $\oplus$-supplemented $(\oplus$-supplemented). Clearly, semisimple modules are completely $I$ - $\oplus$-supplemented. Also, every $I-\oplus$-supplemented hollow module is completely $I$ - $\oplus$ supplemented. The next result provides another example of completely $I$ - $\oplus$-supplemented modules.

Recall that a module $M$ is said to have finite hollow dimension $n \in \mathbb{N}$ if there exists a small epimorphism from $M$ to a direct sum of $n$ hollow modules. We denote this by $h \cdot \operatorname{dim}(M)=n$. It is well known that a module $M$ is hollow if and only if $h \cdot \operatorname{dim}(M)=1$ (see [5, p. 47 and p. 49]).

4.4. Proposition. Let $M=H_{1} \oplus H_{2}$ be a direct sum of hollow submodules $H_{1}$ and $H_{2}$. Then the following statements are equivalent:

(i) $H_{1}$ and $H_{2}$ are $I$ - $\oplus$-supplemented modules;

(ii) The module $M$ is completely $I$ - $\oplus$-supplemented.

Proof. (i) $\Rightarrow$ (ii) Let $L$ be a nonzero direct summand of $M$. If $L=M$, then $L$ is $I$ - $\oplus$-supplemented by Theorem 3.22. Assume that $L \neq M$. Let $K$ be a submodule of $M$ such that $M=L \oplus K$. By $[5,5.4(1)], h \cdot \operatorname{dim}(M)=2=h \cdot \operatorname{dim}(L)+h \cdot \operatorname{dim}(K)$. It follows that $h \cdot \operatorname{dim}(L)=1$ and hence $L$ is a hollow module. Let us prove that $L$ is $I$ - $\oplus$-supplemented. To see this, it suffices to show that $I L=L$ or $I L=\operatorname{Rad}(L)$ by Proposition 3.7. Since $M$ is $I$ - $\oplus$-supplemented, $M / I M \cong(L / I L) \oplus(K / I K)$ is semisimple by Proposition 3.9. As $L$ is a hollow module, $L / I L=0$ or $L / I L$ is simple. Hence $L=I L$ or $L$ is a local module with maximal submodule $I L$. So $I L=L$ or $I L=\operatorname{Rad}(L)$, as required.

(ii) $\Rightarrow$ (i) This is immediate.

\section{Modules over Dedekind domains}

Our purpose in this section is to determine the structure of all $I$ - $\oplus$-supplemented modules and all $\oplus$ - $\delta$-supplemented modules over Dedekind domains.

5.1. Proposition. Let $R$ be a Dedekind domain which is not a field. Then the following assertions are equivalent for an injective $R$-module $M$ :

(i) $M$ is $\oplus$-supplemented;

(ii) $M$ is $I$ - $\oplus$-supplemented for every nonzero ideal $I$ of $R$;

(iii) $M$ is $I$ - $\oplus$-supplemented for some nonzero ideal $I$ of $R$;

(iv) $M$ is $\oplus$ - $\delta$-supplemented.

Proof. (i) $\Rightarrow$ (ii) This follows from Corollary 3.20 since the module $M$ is divisible.

(ii) $\Rightarrow$ (iii) and (iii) $\Rightarrow$ (iv) These are obvious.

(iv) $\Rightarrow$ (i) Since $R$ is a Dedekind domain which is not a field and $M$ is an injective $R$-module, we have $\operatorname{Rad}(M)=M$. The result follows from Proposition 3.13.

Let $R$ be a Dedekind domain which is not a field. If $M$ is an $R$-module, we will denote the sum of all divisible (injective) submodules of $M$ by $d(M)$. It is well known that $d(M)$ is an injective $R$-module. Also, note that if $f$ is an endomorphism of $M$, then $f(d(M))$ is isomorphic to a factor module of $d(M)$. So, $f(d(M))$ is injective as $R$ is a Dedekind domain. Therefore, $f(d(M)) \subseteq d(M)$. It follows that $d(M)$ is a fully invariant submodule of $M$.

5.2. Proposition. Let $R$ be a Dedekind domain which is not a field. Let $I$ be an ideal of $R$ and let $M$ be an $R$-module. Then the following are equivalent:

(i) $M$ is $\oplus$ - $\delta$-supplemented ( $I$ - $\oplus$-supplemented);

(ii) $M$ can be written as $M=M_{1} \oplus M_{2}$ such that $M_{1}$ is injective, $\operatorname{Rad}\left(M_{2}\right) \ll M_{2}$ and both of $M_{1}$ and $M_{2}$ are $\oplus-\delta$-supplemented ( $I-\oplus$-supplemented) modules. 
Proof. (i) $\Rightarrow$ (ii) Let $M_{1}=d(M)$ and let $M_{2}$ be a submodule of $M$ such that $M=M_{1} \oplus M_{2}$. Note that $M_{2}$ has no submodules $X$ with $\operatorname{Rad}(X)=X$. Since $M$ is $\oplus$ - $\delta$-supplemented $(I-\oplus$ supplemented), $M_{1}$ and $M_{2}$ are $\oplus$ - $\delta$-supplemented ( $I-\oplus$-supplemented) by [14, Theorem 2.5] and Proposition 4.3. Moreover, we have $\operatorname{Rad}\left(M_{2}\right) \ll M_{2}$ by Proposition 3.10 .

(ii) $\Rightarrow$ (i) This follows by [14, Theorem 2.2] and Theorem 3.22.

Next, we restrict our investigations about $\oplus$ - $\delta$-supplemented modules and $I$ - $\oplus$-supplemented modules to the case of modules over discrete valuation rings.

5.3. Proposition. Let $M$ be a module over a discrete valuation ring $R$ and let $I$ be an ideal of $R$. Then $M$ is $\oplus-\delta$-supplemented if and only if $M$ is $\oplus$-supplemented. In particular, every $I-\oplus-$ supplemented $R$-module is $\oplus$-supplemented.

Proof. Assume that $M$ is $\oplus$ - $\delta$-supplemented. By Proposition 5.2, $M=M_{1} \oplus M_{2}$ is a direct sum of a $\oplus$ - $\delta$-supplemented injective submodule $M_{1}$ and a submodule $M_{2}$ with $\operatorname{Rad}\left(M_{2}\right) \ll M_{2}$. By Proposition 5.1, $M_{1}$ is $\oplus$-supplemented. In addition, $M_{2}$ is $\oplus$-supplemented by [20, Lemma 2.1] and [12, Proposition A.7]. Therefore, $M$ is $\oplus$-supplemented by [8, Theorem 1.4]. The converse is immediate.

The remaining assertion is obvious.

Let $P$ be a nonzero prime ideal of a Dedekind domain $R$ and let $n$ be a nonzero natural number. We will use the notation $B_{P}(1, \ldots, n)$ to denote the direct sum of arbitrarily many copies of $R / P$, $R / P^{2}, \ldots, R / P^{n}$.

The next result provides a structure theorem for modules over a discrete valuation ring.

5.4. Theorem. Assume that $R$ is a discrete valuation ring with maximal ideal $m$, quotient field $K$ and $Q=K / R$. Let $I$ be an ideal of $R$ and let $M$ be an $R$-module.

(1) If $I=m$ or $I=R$, then the following are equivalent:

(i) $M$ is $I$ - $\oplus$-supplemented;

(ii) $M$ is $\oplus$ - $\delta$-supplemented;

(iii) $M$ is $\oplus$-supplemented;

(iv) $M \cong R^{a} \oplus K^{b} \oplus Q^{c} \oplus B_{m}(1, \ldots, n)$ for some natural numbers $a, b, c$ and $n$.

(2) If $I \notin\{m, R\}$, then the following are equivalent:

(i) $M$ is $I$ - $\oplus$-supplemented;

(ii) $M \cong K^{b} \oplus Q^{c} \oplus(R / m)^{(\Lambda)}$ for some natural numbers $b$ and $c$ and an index set $\Lambda$.

Proof. (1) (i) $\Leftrightarrow$ (iii) By Corollaries 3.18 and 3.19 and Proposition 5.3.

(ii) $\Leftrightarrow$ (iii) By Proposition 5.3.

(iii) $\Leftrightarrow$ (iv) This follows from [12, Proposition A.7].

(2) (i) $\Rightarrow$ (ii) Assume that $M$ is $I$ - $\oplus$-supplemented. By Proposition 5.3, $M$ is $\oplus$-supplemented. Applying [12, Proposition A.7], $M \cong R^{a} \oplus K^{b} \oplus Q^{c} \oplus B_{m}(1, \ldots, n)$ for some natural numbers $a$, $b, c$ and $n$. Since $M / I M$ is semisimple (see Proposition 3.9) and $I \notin\{m, R\}$, we have $a=0$ and for each $1 \leq i \leq n, R /\left(I+m^{i}\right)$ is semisimple. So, for each $1 \leq i \leq n$, we have $I+m^{i}=m$ or $I+m^{i}=R$. Therefore $n=1$ because $I \subseteq m^{2}$. It follows that $B_{m}(1, \ldots, n)=B_{m}(1)$ is semisimple, completing the proof.

(ii) $\Rightarrow$ (i) Note that $K^{b} \oplus Q^{c}$ is an injective $\oplus$-supplemented module by [12, Proposition A.7]. The result follows from Propositions 5.1 and 5.2.

5.5. Remark. Let $R$ be a discrete valuation ring with maximal ideal $m$, quotient field $K$ and $Q=K / R$. Let $I$ be an ideal of $R$. 
(i) Assume that $I \notin\{m, R\}$. Theorem 5.4(2) and [12, Proposition A.7] provide many examples of $\oplus$-supplemented $R$-modules which are not $I$ - $\oplus$-supplemented.

(ii) Note that $[11$, Corollary 4.5$]$ shows that every $\oplus$-supplemented $R$-module is completely $\oplus$ supplemented.

Case 1. Assume that $I \in\{m, R\}$. Then every $I$ - $\oplus$-supplemented $R$-module is completely $I$ - $\oplus$ supplemented by Theorem 5.4.

Case 2. Suppose that $I \notin\{m, R\}$. Let $M$ be an $I$ - $\oplus$-supplemented $R$-module. Then $M=$ $K^{b} \oplus Q^{c} \oplus(R / m)^{(\Lambda)}$ for some natural numbers $b$ and $c$ and an index set $\Lambda$. Let $N$ and $L$ be submodules of $M$ such that $M=N \oplus L$ and let $d(M)$ be the sum of all injective submodules of $M$. It is clear that $d(M)=d(N) \oplus d(L)=K^{b} \oplus Q^{c}$. Then, $d(N) \cong K^{b^{\prime}} \oplus Q^{c^{\prime}}$ for some natural numbers $b^{\prime}$ and $c^{\prime}$ by [2, Corollary 12.7 and Lemma 25.4]. Therefore, $d(N)$ is $I$ - $\oplus$-supplemented by Theorem 5.4. In addition, we have $(R / m)^{(\Lambda)} \cong M / d(M) \cong(N / d(N)) \oplus(L / d(L))$. Hence, $N / d(N)$ is semisimple. Thus, $N / d(N)$ is $I$ - $\oplus$-supplemented. Since $d(N)$ is a direct summand of $N, N$ is $I$ - $\oplus$-supplemented by Theorem 3.22. Consequently, $M$ is completely $I$ - $\oplus$-supplemented

Let $L$ be a submodule of a module $M$. A submodule $K \leq M$ is called a $\delta$-supplement of $N$ in $M$ if $M=L+K$ and $L \cap K \ll_{\delta} K$. The module $M$ is called $\delta$-supplemented if every submodule has a $\delta$-supplement in $M$.

Our next goal is to describe $\oplus$ - $\delta$-supplemented modules and $I$ - $\oplus$-supplemented modules over a nonlocal Dedekind domain $R$. The next proposition shows that every torsion-free $\delta$-supplemented $R$-module is injective. First we prove the following lemma.

5.6. Lemma. Let $L$ be a proper submodule of a module $M$ such that $M / L$ is a cyclic module.

(i) If $K$ is a $\delta$-supplement of $L$ in $M$, then $K=P \oplus R x$, where $P$ is a semisimple projective submodule of $L \cap K$ and $x \in K$. In this case, $R x$ is a $\delta$-supplement of $L$ in $M$.

(ii) If $L$ has a $\delta$-supplement that is a direct summand of $M$, then $L$ has a cyclic $\delta$-supplement that is a direct summand of $M$.

Proof. (i) By assumption, we have $L+K=M$ and $L \cap K \ll_{\delta} K$. Thus, $M / L \cong K /(L \cap K)$ is cyclic. Let $x \in K$ such that $K=(L \cap K)+R x$. Since $L \cap K \ll_{\delta} K$, there exists a semisimple projective submodule $P$ of $L \cap K$ such that $K=P \oplus R x$ by Lemma 2.1. Note that $L \cap K=L \cap(P \oplus R x)=$ $P \oplus(L \cap R x) \ll{ }_{\delta} P \oplus R x$. By Lemma 2.2(iv), we have $P \ll_{\delta} P$ and $L \cap R x \ll_{\delta} R x$. Therefore $P$ is a semisimple projective module by [15, Lemma 2.9]. Also, note that $L+R x=M$. It follows that $R x$ is a $\delta$-supplement of $L$ in $M$.

(ii) follows from (i).

5.7. Proposition. Assume that $R$ is a Dedekind domain which is not local. Let $K$ denote the quotient field of $R$. If $M$ is a $\delta$-supplemented $R$-module, then $M / T(M) \cong K^{(\Lambda)}$ for some index set $\Lambda$.

Proof. Assume that $M$ has a maximal submodule $L$ such that $T(M) \subseteq L$. Since $M$ is $\delta$-supplemented, there exists a cyclic submodule $W$ of $M$ such that $M=L+W$ and $L \cap W \ll_{\delta} W$ (see Lemma 5.6). Let $A$ be an ideal of $R$ such that $W \cong R / A$. Since $W$ is not contained in $L, W$ is not a torsion module. So $A=0$ and $W \cong{ }_{R} R$. Thus, $W$ is an indecomposable $R$-module. Hence $L \cap W \ll W$ by Proposition 2.3. Since $W /(L \cap W) \cong M / L$, we conclude that $W$ is a local submodule of $M$. This contradicts the fact that $R$ is not a local ring. It follows that $\operatorname{Rad}(M / T(M))=M / T(M)$. Hence, the module $M / T(M)$ is injective. So there exists an index set $\Lambda$ such that $M / T(M) \cong K^{(\Lambda)}$ by $[9$, Lemma 2.1].

5.8. Proposition. Assume that $R$ is a Dedekind domain which is not local. If $M$ is a $\oplus-\delta$ supplemented $R$-module with $\operatorname{Rad}(M) \ll M$, then $M$ is a torsion module. 
Proof. Since $M$ is $\oplus$ - $\delta$-supplemented, there exist submodules $A$ and $B$ of $M$ such that $M=A \oplus B=$ $T(M)+B$ and $T(M) \cap B \ll \delta B$. Since $T(M)=T(A) \oplus T(B)$, we have $M=T(A) \oplus B$ and $T(M)=T(A) \oplus(T(M) \cap B)$. Hence $T(A)=A$ and $T(B)=T(M) \cap B$. So, $T(B) \ll \delta B$. By Proposition 2.6, we have $T(B) \ll B$. Note that $M / T(M) \cong B / T(B)$ is divisible by Proposition 5.7. It follows that for every nonzero element $r \in R$, we have $r B+T(B)=B$. So, $r B=B$ for every $0 \neq r \in R$. This implies that $B$ is a divisible module, that is, $\operatorname{Rad}(B)=B$ (see $[9$, Lemma 2.1]). But $\operatorname{Rad}(B) \ll B$ since $\operatorname{Rad}(M) \ll M$. Then $B=0$ and $M=A$ is a torsion module, as required.

5.9. Proposition. Assume that $R$ is a nonlocal Dedekind domain. If $M$ is a $\oplus-\delta$-supplemented $R$-module, then $M$ is a torsion module.

Proof. By Proposition 5.2, $M=M_{1} \oplus M_{2}$ is a direct sum of $\oplus$ - $\delta$-supplemented submodules $M_{1}$ and $M_{2}$ such that $\operatorname{Rad}\left(M_{1}\right)=M_{1}$ and $\operatorname{Rad}\left(M_{2}\right) \ll M_{2}$. By Proposition 5.1, $M_{1}$ is $\oplus$-supplemented. So, $M_{1}$ is a torsion module by [12, Proposition A.8]. Moreover, $M_{2}$ is a torsion module by Proposition 5.8. Therefore $M$ is a torsion module, as required.

5.10. Corollary. Assume that $R$ is a nonlocal Dedekind domain. An $R$-module $M$ is $\oplus-\delta$-supplemented if and only if $M$ is $\oplus$-supplemented.

Proof. This follows easily from Propositions 3.14 and 5.9.

5.11. Remark. Combining Proposition 5.3, Corollary 5.10 and [12, Propositions A.7 and A.8], we obtain the structure of $\oplus$ - $\delta$-supplemented modules over Dedekind domains.

5.12. Lemma. Assume that $R$ is a Dedekind domain which is not local. Let $P$ be a maximal ideal of $R$ and let $i$ be a nonzero natural number. Then:

(i) $I+P=P$ if and only if $I \subseteq P$.

(ii) If $i \geq 2$, then $I+P^{i}=P$ if and only if $I \subseteq P$ and $I \nsubseteq P^{2}$.

(iii) $I+P^{i}=R$ if and only if $I \nsubseteq P$.

Proof. (i) and (iii) are immediate.

(ii) $(\Rightarrow)$ This is obvious.

$(\Leftarrow)$ By hypothesis, we have $I=P I^{\prime}$, where $I^{\prime}$ is an ideal of $R$ which is not contained in $P$ (see $\left[13\right.$, Theorem 6.14]). Since $I^{\prime}+P^{(i-1)}=R$, we see that $P I^{\prime}+P^{i}=P$. Hence, $I+P^{i}=P$.

Let $M$ be a module over a Dedekind domain $R$ and let $P$ be a nonzero prime ideal of $R$. We will denote by $M_{P}$ the set $\left\{x \in M \mid P^{n} x=0\right.$ for some integer $\left.n \geq 0\right\}$ which is called the $P$-primary component of $M$. Note that if $M$ is a torsion $R$-module, then $M$ is a direct sum of its $P$-primary components. Let $K$ be the quotient field of $R$. We will denote by $R\left(P^{\infty}\right)$ the $P$-primary component of the torsion $R$-module $K / R$. It is well known that $R\left(P^{\infty}\right)$ is a hollow module (see [9, Lemma $2.4])$.

The next result describes the structure of $I$ - $\oplus$-supplemented modules over nonlocal Dedekind domains. Recall that a module $M$ is 0 - $\oplus$-supplemented if and only if $M$ is semisimple (see Remark $3.3(\mathrm{ii}))$.

5.13. Theorem. Assume that $R$ is a nonlocal Dedekind domain. Let $I$ be a nonzero ideal of $R$. Then the following assertions are equivalent for an $R$-module $M$ :

(i) $M$ is $I$ - $\oplus$-supplemented;

(ii) $M$ is torsion and every $P$-primary component of $M$ is $I$ - $\oplus$-supplemented;

(iii) $M$ is torsion and for every nonzero prime ideal $P$ of $R$, there exist natural numbers a and $n$ such that $M_{P} \cong\left(R\left(P^{\infty}\right)\right)^{a} \oplus B_{P}(1, \ldots, n)$ with $n=1$ if $I \subseteq P^{2}$. 
Proof. (i) $\Leftrightarrow$ (ii) It is well known that for every nonzero prime ideal $P$ of $R, M_{P}$ is a fully invariant submodule of $M$. The result follows from Propositions 3.24, 4.3 and 5.9.

(ii) $\Rightarrow$ (iii) Let $P$ be a nonzero prime ideal of $R$. Since $M_{P}$ is $I$ - $\oplus$-supplemented, $M_{P}$ is $\oplus$ supplemented by Corollary 5.10. Thus, there exist natural numbers $a$ and $n$ such that $M_{P} \cong$ $\left(R\left(P^{\infty}\right)\right)^{a} \oplus B_{P}(1, \ldots, n)$ by $[12$, Propositions A.7 and A.8]. Let $1 \leq i \leq n$. Since $M / I M$ is semisimple (see Proposition 3.9), $\left(R / P^{i}\right) /\left(\left(I+P^{i}\right) / P^{i}\right) \cong R /\left(I+P^{i}\right)$ is semisimple. As $R / P^{i}$ is a local $R$-module, we have $I+P^{i}=R$ or $I+P^{i}=P$. Note that if $I \subseteq P^{2}$ and $i \geq 2$, then $I+P^{i} \subseteq P^{2}$. In this case we have $I+P^{i} \neq R$ and $I+P^{i} \neq P$. This shows that $I \subseteq P^{2}$ forces $n=1$.

(iii) $\Rightarrow$ (ii) Let $P$ be a nonzero prime ideal of $R$. Note that $M_{P}$ and $\left(R\left(P^{\infty}\right)\right)^{a}$ are $\oplus$-supplemented by [12, Propositions A.7 and A.8]. We divide the rest of the proof into three cases:

Case 1. Assume that $I \subseteq P^{2}$. By hypothesis, $n=1$. Therefore $B_{P}(1, \ldots, n)=B_{P}(1)$ is semisimple. Hence $M_{P} \cong\left(R\left(P^{\infty}\right)\right)^{a} \oplus B_{P}(1)$ is $I$ - $\oplus$-supplemented (see Proposition 5.1 and Theorem $3.22)$.

Case 2. Suppose that $I \nsubseteq P^{2}$ and $I \nsubseteq P$. Then, $I M_{P}=M_{P}$ by Lemma 5.12(iii). Therefore, $M_{P}$ is $I$ - $\oplus$-supplemented by Corollary 3.18 .

Case 3. Assume that $I \nsubseteq P^{2}$ and $I \subseteq P$. In this case we have $I M_{P}=P M_{P}$ by Lemma 5.12. Applying Corollary 3.19, we conclude that $M_{P}$ is $I$ - $\oplus$-supplemented. This completes the proof.

5.14. Remark. Let $I$ be an ideal of a nonlocal Dedekind domain $R$. Using Theorem $5.13,[17$, Theorem 1] and an analysis similar to that in Remark 5.5, we conclude that every $I$ - $\oplus$-supplemented $R$-module is completely $I$ - $\oplus$-supplemented.

\section{References}

[1] Alkan, M., Nicholson, W. K. and Özcan, A. Ç. A generalization of projective covers, J. Algebra 319, 4947-4960, 2008.

[2] Anderson, F. W. and Fuller, K. R. Rings and Categories of Modules (Springer-Verlag, New-York, 1992).

[3] Atiyah, M. F. and Macdonald, I. G. Introduction to Commutative Algebra (Addison-Wesley, London, 1969).

[4] Büyükaşik, E. and Lomp, C. When $\delta$-semiperfect rings are semiperfect, Turkish J. Math. 34, 317-324, 2010.

[5] Clark, J., Lomp, C., Vanaja, N. and Wisbauer, R. Lifting Modules. Supplements and Projectivity in Module Theory (Frontiers in Mathematics, Birkhäuser, Basel, 2006).

[6] Ecevit, Ş., Koşan, M. T. and Tribak, R. Rad- $\oplus$-supplemented modules and cofinitely Rad- $\oplus$ supplemented modules, Algebra Colloq. 19 (4), 637-648, 2012.

[7] Generalov, A. I. $\omega$-cohigh purity in the category of modules, Math. Notes 33, 402-408, 1983; translation from Mat. Zametki 33, 785-796, 1983.

[8] Harmanci, A., Keskin, D. and Smith, P. F. On $\oplus$-supplemented modules, Acta Math. Hungar. 83 (1-2), 161-169, 1999.

[9] Hausen, J. Supplemented modules over Dedekind domains, Pacific J. Math. 100 (2), 387-402, 1982.

[10] Idelhadj, A. and Tribak, R. A dual notion of CS-modules generalization, in: Algebra and Number Theory (Fez) (M. Boulagouaz and J.-P. Tignol, eds.), Lecture Note of Pure and Appl. Math. 208 (Marcel Dekker, New York, 2000), 149-155.

[11] Idelhadj, A. and Tribak, R. On some properties of $\oplus$-supplemented modules, Internat. J. Math. Math. Sci. 69, 4373-4387, 2003.

[12] Mohamed, S. H. and Müller, B. J. Continuous and Discrete Modules, London Math. Soc. Lecture Note Ser. 147 (Cambridge Univ. Press., Cambridge, 1990).

[13] Sharpe, D. W. and Vámos, P. Injective Modules Cambridge Tracts in Mathematics and Mathematical Physics 62 (Cambridge University Press, London, 1972).

[14] Talebi, Y. and Talaee, B. Generalizations of $D_{11}$ and $D_{11}^{+}$modules, Asian-European J. Math. 2 (2), 285-293, 2009. 
[15] Tribak, R. On $\delta$-local modules and amply $\delta$-supplemented modules, J. Algebra Appl. 12 (2), 1250144 (14 pages), 2013.

[16] Wang, Y. A generalization of supplemented modules, arXiv:1108.3381v1 [math.RA].

[17] Warfield Jr., R. B. A Krull-Schmidt theorem for infinite sums of modules, Proc. Amer. Math. Soc. 22, 460-465, 1969.

[18] Wisbauer, R. Foundations of Module and Ring Theory (Gordon and Breach, Philadelphia, 1991).

[19] Zhou, Y. Generalizations of perfect, semiperfect, and semiregular rings, Algebra Colloq. 7 (3), 305-318, 2000.

[20] Zöschinger, H. Komplementierte moduln über Dedekindringen, J. Algebra 29, 42-56, 1974. 